\title{
Spinal Primitive Neuroectodermal Tumor (PNET) in a 64-year-old Male Treated with Surgery, Radiotherapy, and Chemotherapy: A Case Report
}

Ophel S. Gantuangco, MD and Mary Ondinee Manalo-Igot, MD

Asian Hospital and Medical Center

\begin{abstract}
Primitive neuroectodermal tumors (PNETs) are a heterogeneous group of malignant neoplasms found primarily in childhood and early adulthood. In this paper, we described the case of a 64-year-old male with primary spinal PNET, successfully treated with surgery, craniospinal radiotherapy, and concurrent chemotherapy.

This is the case of a 64-year-old male who presented with a 2-month history of bilateral lower extremity weakness and numbness associated with urinary and bowel incontinence. Work-up was done, and the spine's plain magnetic resonance imaging (MRI) revealed a heterogeneously enhancing intradural lesion with an extradural component at the right T9/T10 level, causing mild to moderate cord compression. The patient underwent laminectomy and gross total resection of the said tumor. Histology and immunohistochemistry were consistent with a primitive neuroectodermal tumor of the spine. The tumor recurred three months after the surgery, and the patient was then referred for radiation therapy with concurrent chemotherapy. Repeat spinal MRIs with three- to six-month intervals after treatment showed no tumor recurrence as of August 2021.
\end{abstract}

Primary spinal PNETs are rarely found in adults, especially in the elderly. These tumors currently have no recommendations or guidelines regarding their management. Thus, most cases are presently being managed based on studies on children and central nervous system (CNS) PNETs.

This paper presented a case of a successfully treated primary spinal PNET in the elderly. The management was primarily based on studies done on that of the pediatric population and CNS PNETs.

Keywords: case report, PNET, peripheral spinal PNET, peripheral primitive neuroectodermal tumor

\section{INTRODUCTION}

Primitive neuroectodermal tumors (PNETs) are a group of aggressive tumors primarily found in children and young adults. These tumors can be classified as central PNETs (cPNETs) or peripheral PNETs (pPNETs). They can be differentiated through CD99 immunopositivity and the presence of $(11 ; 22)$ (q24;q12) chromosomal translocations

Presented as an E-poster in the Asian Oncology Society's $1^{\text {st }}$ Virtual Conference on November 27 - December 2, 2020.

Presented and won $2^{\text {nd }}$ place in the Philippine Society of Medical Oncology's 33rd Annual Convention Oral Research Presentation on October 23-25, 2020.

Presented as an E-poster in the Philippine College of Physicians $50^{\text {th }}$ Annual Convention on August 23-26, 2020.

Corresponding author: Ophel S. Gantuangco, MD

Asian Hospital and Medical Center

2205 Civic Drive, Alabang, Muntinlupa City, Philippines

Email: osgantuangco@gmail.com on pPNETs. ${ }^{1}$ PNETs of the central nervous system (CNS) have been reported in several locations, including the cerebrum, cerebellum, brainstem, pineal gland, spinal cord, and peripheral nerves. Primary pPNETs of the spine are rare, representing only less than $1 \%$ of primary spinal tumors in an extensive series of 430 patients. ${ }^{2,3}$ Adult PNET itself accounts for only under $0.5 \%$ of primary CNS malignancies. ${ }^{4}$

Since Hart and Earle's first description of the condition in 1973, roughly a hundred cases of PNET have been documented. It has been controversial for over a decade, as diagnosis remains difficult and no effective treatment 
has been identified..$^{5}$ In a meta-analysis by Saeedinia et al., among 107 cases of primary intraspinal pPNET, 53 cases were adults, with only one elderly case reported aged 70 years old. ${ }^{6}$ Although found to be the second most common malignant neoplasm in childhood, when PNETs primarily present in the spine, it is relatively rare in all age groups. Data on these tumors in the literature are limited to case reports. ${ }^{7}$ Histologically, the PNET cells exhibit a primitive, poorly differentiated morphology with varying degrees of pleomorphism and occasional evidence of neuroectodermal differentiation. ${ }^{2}$ In this paper, we presented a rare case of primary spinal PNET in an elderly patient, successfully treated with surgery, craniospinal radiotherapy, and concurrent chemotherapy. The patient remained free of the disease as of August 2021. Consent was obtained from the patient to discuss his case through a case report, and strict anonymity will be maintained.

\section{CASE PRESENTATION}

This is the case of a 64-year-old male who presented with bilateral lower extremity weakness and numbness for two months and a sudden onset of a shooting pain on the lower back, radiating to both lower extremities in May of 2017. Symptoms were also associated with urinary and bowel incontinence. The patient is a known hypertensive and diabetic with fair compliance to his maintenance medications. He is a 24-pack-year smoker who stopped smoking in 2016. No family history for malignancy was elicited. Physical examination revealed that the last normal sensory level was at T9, and the motor strength of both lower extremities was at $3 / 5$. Other findings from the rest of the physical examination were unremarkable. The consult was done at a local private hospital. He underwent an MRI of the spine, which showed a heterogeneously enhancing intradural lesion with an extradural component at the right T9/T10 level measuring $7.0 \times 2.0 \times 3.0 \mathrm{~cm}$ causing mild to moderate cord compression (Figure 1).To rule out a metastatic tumor, brain, and thoracic MRI, chest, and whole abdominal CT-scans were done, which turned out to be unremarkable.

Because of the patient's symptoms brought about by the mass, the patient was appraised for laminectomy and tumor excision biopsy. However, he opted to have this done at a government hospital due to financial constraints. The patient underwent T8-T10 laminectomy with gross total excision of the tumor. Thereafter, the patient claimed minor improvement with the motor strength and sensation of his lower extremities. The evaluation showed a small round cell tumor with highly vascular stroma and pseudorosette formation by tumor cells on routine histopathology.

Immunohistochemistry stains were positive for synaptophysin, a broad-spectrum neuroendocrine marker, and CD99, which is highly specific for PNET and was negative for chromogranin, LCA, CD34, S100, pan-cytokeratin, CAM5.2, desmin, and WT-1. The final histopathologic diagnosis was a peripheral primitive neuroectodermal tumor of the spine.

In both primary intraspinal and CNS PNETs, chemotherapy has improved 1- and 2-year survival rates after total or subtotal tumor resection; thus, the patient was advised adjuvant treatment but was lost to follow-up. ${ }^{2}$

Three months after the surgery (October 2017), the patient felt the progressive weakness of his lower extremities, now with only a motor strength of $2 / 5$ bilaterally. On repeat
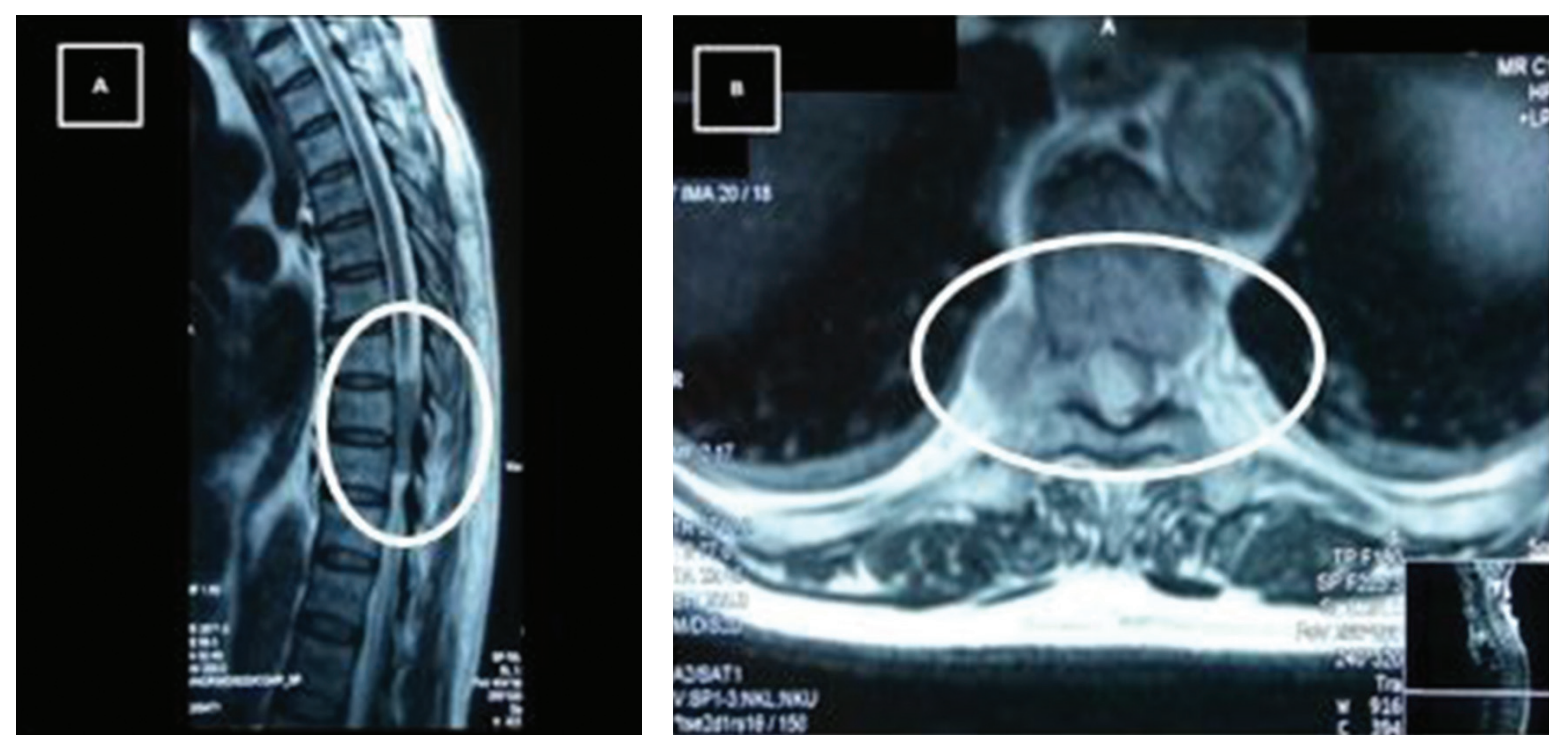

Figure 1. Magnetic Resonance Imaging (MRI) of the Thoracic Spine showed (A) a heterogeneously enhancing intradural lesion with (B) an extradural component at the right T9/T10 level measuring $7.0 \times 2.0 \times$ $3.0 \mathrm{~cm}$ causing mild to moderate cord compression. 


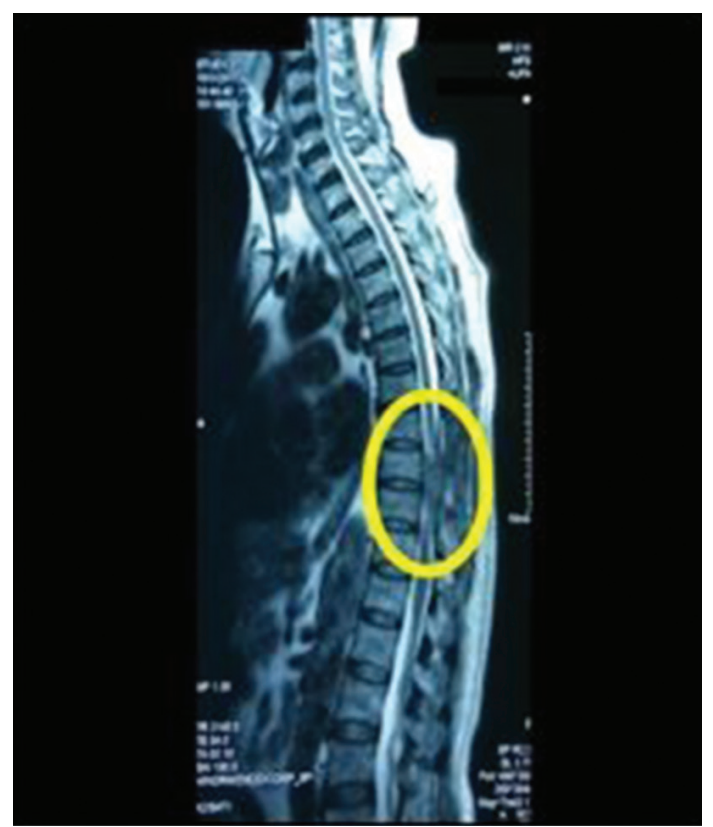

Figure 2. Repeat thoracic spine MRI of the patient case done three months after surgery (October 2017) showed recurrence of the mass at T9/T10 level measuring 3.5 $\times 2.8 \times 2.5 \mathrm{~cm}$.

MRI of the thoracic spine, there was a recurrence of the mass at the T9/T10 level measuring approximately $3.5 \times 2.8 \times 2.5$ cms causing mild to moderate cord compression (Figure 2).

Re-excision was advised with these findings, but the patient did not want to undergo another surgery. He opted to undergo radiation therapy concurrent with chemotherapy instead. The patient was then referred to us for further management. He underwent a cranial and whole spine MRI before starting the treatment since these tumors tend to be located in multiple areas of the neuraxis. Results of such tests showed no involvement of other sites.

The patient received craniospinal irradiation (CSI) via conventional planning. The whole brain and the whole spine (down to the end of the thecal sac) received $36 \mathrm{~Gy}$ in 20 fractions. An additional $18 \mathrm{~Gy}$ in 10 fractions was delivered to boost the residual tumor at T9/T10 to a cumulative 54 Gy in 30 fractions. Concurrent chemotherapy was given as follows: cisplatin at $50 \mathrm{mg} / \mathrm{m}^{2}$ on day 1 ; vincristine at $1 \mathrm{mg}$ bolus on days 2, 8, and 15; and cyclophosphamide at 750 $\mathrm{mg} / \mathrm{m}^{2}$ on days 22 and 23 . The doses given were slightly lower than published doses as they were dose reduced by $30-40 \%$ because of the patient's age and functional status. Granulocyte-colony stimulating factor (G-CSF) support was given weekly. There was an unplanned one-week treatment interruption during the middle of the regimen because of severe weakness attributed to dehydration and severe hypokalemia as side effects of cisplatin. He completed treatment without difficulty after this.

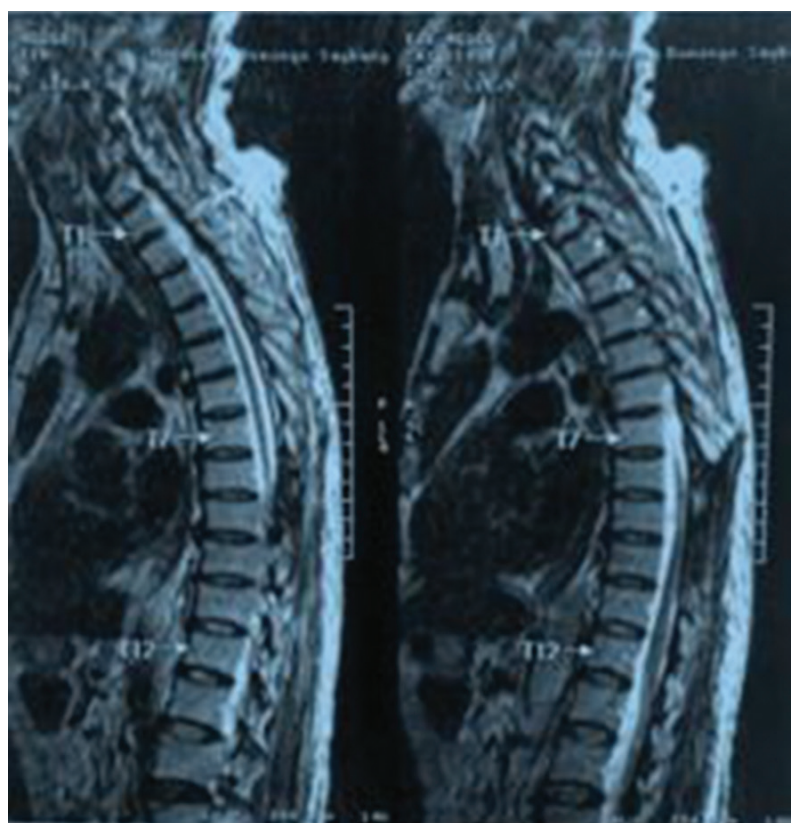

Figure 3. Whole spine MRI of the patient case four months after craniospinal irradiation and concurrent chemotherapy (April 2018) showed laminectomy defects at T9 and T10 with no evidence of paraspinal enhancing lesion.

Repeat whole spine MRIs of the patient at 3-6-month intervals (April 2018, October 2018, and May 2019) showed no evidence of tumor recurrence (Figure 3). Cranial MRIs also did not show any evidence of disease.

As of August 2021, the motor strength of both of his lower extremities is at 3-4/5. Hypoesthesia from T9 below was also noted and reported with a $40 \%$ sensory deficit. Although he did not regain his full strength even after chemoradiation therapy, he has continued his physical therapy and rehabilitation exercises and improved. His bowel and urinary incontinence, however, were unresolved.

\section{DISCUSSION}

Primary PNETs found in the spine are rare lesions. The majority of spinal PNETs are the result of the subarachnoidal spread of tumors in the neuraxis. ${ }^{8}$ Before the diagnosis of primary spinal PNET can be confirmed, the more common "drop" metastases of intracranial origin must be excluded. ${ }^{9}$ This is the main reason why the patient underwent cranial and whole spine MRI before starting craniospinal irradiation (CSI) and concurrent chemotherapy.

According to a meta-analysis by Saeedinia et al., they reviewed 107 cases of intraspinal peripheral PNETs (pPNETs) reported in the literature. ${ }^{6}$ The average age of occurrence for primary intraspinal pPNET was 22 years of age. Among these 107 patients, $66 \%$ were males. The average duration of symptoms before diagnosis was 4.52 months. 
Spinal PNET in the Elderly

Table 1. Summary of the cases of primary spinal primitive neuroectodermal tumors (PNET)-Ewing's sarcoma family tumors in the elderly (>60 years old) reported in the literature

\begin{tabular}{|c|c|c|c|c|c|c|}
\hline Author & Year & $\begin{array}{c}\text { Age at } \\
\text { Diagnosis/Sex }\end{array}$ & Location of Tumor & Treatment & $\begin{array}{c}\text { Follow-up/ } \\
\text { Survival (month) }\end{array}$ & Outcome \\
\hline Fabre et al. ${ }^{10}$ & 2006 & $70 / \mathrm{M}$ & Cauda Equina & $\mathrm{STR} / \mathrm{RT} / \mathrm{CT}$ & 12 & Alive \\
\hline Marwin et al..$^{9}$ & 2002 & $69 / \mathrm{M}$ & Cervical-thoracic, intra-, and extra-medullary & $\mathrm{STR} / \mathrm{RT}$ & 3 & Dead \\
\hline Shimo-sawa et al. ${ }^{8}$ & 2011 & $63 / \mathrm{M}$ & Conus medullaris from L1 to $\mathrm{L} 2$ & TTR/RT/CT & 25 & Dead \\
\hline
\end{tabular}

M: Male; STR: Sub-total tumor resection; TTR: Total tumor resection; CT: Chemotherapy; RT: Radiotherapy

The incidence in the lumbar region is twice as much as in the thoracic and cervical regions. Among these patients, 64 cases received both radiotherapy and chemotherapy after total or subtotal tumor resection. They had a better one-year survival rate than those who did not receive chemoradiation therapy. Based on the studies gathered in the meta-analysis, among the 107 cases reported, only one patient was elderly or above 60 years old, having an incidence of only $0.93 \%$ in all cases of intraspinal pPNET in their meta-analysis. In another study by Shimosawa et al., a total of two elderly patients were found during their literature search for primary spinal PNETs. ${ }^{8}$

Table 1 summarizes the cases of primary spinal PNETs in the elderly gathered from our literature search. This case makes it the fourth case of primary spinal PNET in the elderly so far, to the best of our knowledge.

MRI of the spine is the investigation of choice. MRI provides excellent soft-tissue imaging within the spinal column. Still, findings vary from one patient to another radiographically and are generally not helpful in differentiating PNET from other primary spinal tumors. Thus, the definitive diagnosis of PNET can only be made from a limited biopsy or radical resection of tumor tissue. Furthermore, histological analysis alone is not sufficient for diagnosis, and immunohistochemistry is required. ${ }^{2}$ Histopathologically, PNETs are undifferentiated, small, round-cell tumors with hyperchromatic nuclei and features of neural differentiation, which typically form Homer Wright rosettes. Immunohistochemically, tumor cells are positive for CD99 and HBA71 which are products of the microneme protein 2 (MIC2) gene. Immunoreactivity for synaptophysin, NSE, S100, and neurofilament indicate neuroectodermal differentiation. ${ }^{6}$

In the case of our patient, the diagnosis of a spinal tumor was diagnosed through a thoracic MRI. When the tumor was resected, it was sent for biopsy, and the histopathologic features and immunostaining were consistent with a peripheral primitive neuroectodermal tumor, establishing the patient's diagnosis.

The treatment of spinal PNETs remains vague and unidentified because of the lack of evidence and rarity of the condition. Currently, spinal PNET is treated in the same way as other CNS PNETs and extraspinal PNETs. The mainstay of treatment remains surgical for both decompression and diagnosis and should be performed with total resection to remove as much tumor as possible. ${ }^{8}$ The patient underwent gross total resection of the tumor two months after his presenting signs and symptoms. However, PNET is a highly aggressive cancer and has a postoperative recurrence rate of $90 \% .^{11}$ True enough, the patient's tumor recurred within three months after his surgery when he experienced progression of bilateral lower extremity weakness.

In the Adult PNET Guidelines by the British NeuroOncology Society/National Cancer Action Team (NCAT) Rare Tumour Guidelines published in 2011, PNETs are characterized by radiosensitivity and chemosensitivity. ${ }^{4}$ While there is limited clinical data to define the optimal treatment strategy for PNETs in adults, management strategies for children are based on the results of a series of consecutive European and North American multiinstitutional trials using upfront craniospinal irradiation and the routine use of concurrent and adjuvant chemotherapy.

Postoperative radiotherapy remains a cornerstone in the management of primary CNS PNETs to achieve longterm tumor control. ${ }^{2}$ During the patient's follow-up, he was initially advised for tumor re-excision but opted to have CSI and concurrent chemotherapy. Due to the aggressiveness of these tumors, the prognosis is still poor, and the disease-free survival is approximately $45 \%$ at seven years. In PNETs found in the brain, surveillance imaging could be considered since currently available salvage strategies can achieve long-term remission with localized recurrences amenable to further surgical resection and repeat radiotherapy. However, it is not recommended due to the lack of evidence of a patient deriving benefit from regular spinal imaging. It should only be performed in case of clinically suspicious symptoms or evidence of a local recurrence. ${ }^{2}$ Nevertheless, our patient is having routine spinal imaging every 3-6 months since he has not yet fully recovered from his symptoms, making it hard to establish recurrence without imaging. As of August 2021, the patient remains disease-free with a total progressionfree survival of 44 months.

\section{CONCLUSION}

Primary spinal PNET is a rare condition that can present with acute neurological deficits. It is rarely found in adults, especially in the elderly, and because of its low incidence, the treatment is based on PNETs found on the CNS and in children, as demonstrated in this case report. Despite its 
radiosensitivity and chemosensitivity, the prognosis is still poor. There are no current recommendations or guidelines regarding management and surveillance, which would be a good topic for research and prospective trials. A study on its molecular pathogenesis can also be helpful to identify potential molecular therapeutic targets in the future.

\section{Statement of Authorship}

Both authors contributed in the conceptualization of work; acquisition and analysis of data; drafting and revising; and final approval of the version to be published.

\section{Author Disclosure}

Both authors declared no conflicts of interest.

\section{Funding Source}

This study has no funding support.

\section{REFERENCES}

1. Kampman WA, Kros JM, De Jong TH, Lequin MH. Primitive Neuroectodermal Tumors (PNETs) Located in the Spinal Canal; the Relevance of Classification as Central or Peripheral PNET. J Neurooncol. 2006;77:65-72.

2. Eghbal K, Dehghanian AR and Ghaffarpasand F. Lumbosacral Epidural Primitive Neuroectodermal Tumor (PNET): Case Report and Literature Review. Turk Neurosurg. 2018;28(6):1005-1008.
3. Engelhard HH, Villano JL, Porter KR, Stewart AK, Barua M, Barker FG, et al. Clinical Presentation, Histology, and Treatment in 430 Patients with Primary Tumors of the Spinal Cord, Spinal Meninges, or Cauda Equina. J Neurosurg Spine. 2010 Jul;13(1):67-77.

4. British Neuro-Oncology Society/NCAT Rare Tumour Guidelines, Guidelines on the Diagnosis and Management of Adult PNETs [Internet]. June 2011 [cited 2021 Aug]. Available from: https://bnos. org.uk

5. Meng X, He S. Primitive Neuroectodermal Tumor in the Spinal Canal: A Case Report. Oncol Lett. 2015 Apr;9(4):1934-6.

6. Saeedinia S, Nouri M, Alimohammadi M, Moradi H, Amirjamshidi A. Primary Spinal Extradural Ewing's Sarcoma (Primitive Neuroectodermal Tumor): Report of a Case and Meta-analysis of the Reported Cases in the Literature. Surg Neurol Int. 2012;3: 55.

7. Khmou M, Malihy A, Lamalmi N, Rouas L, Alhamany Z. Peripheral Primitive Neuroectodermal Tumors of the Spine: A Case Report and Review of the Literature. BMC Res Notes. 2016 Sep 9;9(1):438.

8. Shimosawa H, Matsumoto M, Yabe H, Mukai M, Toyama $\mathrm{Y}$, Morioka H. Primary Primitive Neuroectodermal Tumor of the Conus Medullaris in an Elderly Patient: A Case Report and Review of the Literature. Case Rep Oncol. 2011 May-Aug;4(2):267-74.

9. Mawrin C, Synowitz HJ, Kirches E, Kutz E, Dietzmann K, Weis S. Primary Primitive Neuroectodermal Tumor of the Spinal Cord: Case Report and Review of the Literature. Clin Neurol Neurosurg. 1 Jan 2002;104(1):36-40.

10. Fabre E, Guillevin R, Chretien F, Le Guerinel C, Duffau H. Peripheral Primitive Neuroectodermal Tumor of the Cauda Equina in an Elderly Patient. Case Report. J Neurosurg Spine. 2006 Jul;5:68-71.

11. Shah JP, Jelsema J, Bryant CS, Ali-Fehmi R, Malone JM Jr. Carboplatin and Paclitaxel Adjuvant Chemotherapy in Primitive Neuroectodermal Tumor of the Uterine Corpus. Am J Obstet Gynecol. 2009 Feb;200(2):e6-e9

\section{The Acta Medica Philippina is now accepting limited advertising for its front and back cover (colored), as well as for available spaces in some of its pages, as appropriate. For inquiries and submission of proposals, please email us at actamedicaphilippina.upm@up.edu.ph}

